\title{
Available Tools to Facilitate Early Patient Access to Medicines in the EU and the USA: Analysis of Conditional Approvals and the Implications for Personalized Medicine
}

\author{
Lada Leyens $^{\mathrm{a}}$ Étienne Richer ${ }^{\mathrm{b}} \quad \varnothing_{\text {yvind Melien }}{ }^{\mathrm{C}}$ Wolfgang Ballensiefen ${ }^{\mathrm{d}}$ \\ Angela Brand ${ }^{a}$ \\ ${ }^{a}$ Maastricht University, Maastricht, The Netherlands; ${ }^{b} \mathrm{CIHR}$ - Institute of Genetics, Montreal, Que., Canada;

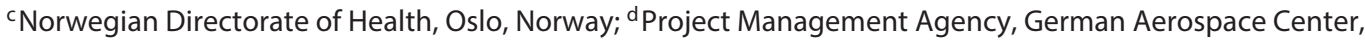 \\ Bonn, Germany
}

\section{Key Words}

Early patient access $\cdot$ Conditional approval $\cdot$ Accelerated approval $\cdot$ Personalized medicine $\cdot$ Drug development .

Regulation · European Medicines Agency · Food and Drug

Administration $\cdot$ Health technology assessment

\begin{abstract}
Scientific knowledge and our understanding of the human body and diseases have limited any possible treatment tailoring to each patient. The technological advances enabling the integration of various data sets (e.g. '-omics', microbiome, epigenetics and environmental exposure) have facilitated a greater understanding of the human body, the molecular basis of disease and all the factors influencing disease onset, progression and response to treatment, thereby ushering in the era of personalized medicine. We evaluate the regulatory approaches available to facilitate early patient access to efficacious and safe compounds in the EU and the USA in order to make more informed recommendations in the future as to the gaps in regulations for early patient access. An in-depth analysis of conditional approvals (EU) and accelerated approvals (USA) is performed based on the pub-
\end{abstract}

licly available information (European public assessment reports and a summary review of products approved under both programmes). The types of product, indications, time to approval and type of evidence submitted were analysed. Between 2007 and early 2015, 17 products were conditionally approved in the EU and 25 in the USA, most of them in the area of oncology and based on evidence from phase II clinical trial data. Early approval of promising products based on data from early phases of development is already possible in the EU and the USA. Some of the improvements could entail implementing a rolling assessment of evidence in Europe and extending the scope of early dialogues.

(c) 2015 S. Karger AG, Basel

\section{Introduction}

Scientific knowledge and our understanding of the human body and diseases limit any possible treatment tailoring to each patient. Doctors have always used precise medicine, i.e. as precise as the knowledge in medicine allowed them to do, and they continue to do so. What is different in the so-called 'new era of personalized medi-

\section{KARGER 125}

(c) 2015 S. Karger AG, Base

$1662-4246 / 15 / 0185-0249 \$ 39.50 / 0$

E-Mail karger@karger.com

www.karger.com/phg
Lada Leyens

Maastricht University

Universiteitssingel 40

NL-6229 ER Maastricht (The Netherlands)

E-Mail lada.leyens@ maastrichtuniversity.nl 
cine' are the great advances in '-omic' sciences (genomics, transcriptomics, proteomics, metabolomics, etc.) and in Information and Communication Technologies in the previous 2 decades, and consequently in our understanding of the molecular mechanisms of diseases. The staggering fall in the costs of gene sequencing and genotyping together with other technological advances enabling the integration of various data sets (e.g. microbiome, epigenetics and environmental exposure) have facilitated a greater understanding of the human body, the molecular basis of disease and all the factors influencing disease onset, progression and response to treatment $[1,2]$.

In drug development, there has been a paradigm shift in the past 30 years. We have moved from an anatomical classification, a 'one-size-fits-all' concept with large disease groups (e.g. all cancers classified under one disease group) and blockbuster medicines that are used to treat millions of patients, through a histological classification of disease to a molecular classification [3] and the stratification into smaller sub-groups of conditions and patients, each treated with different drugs targeted at specific genetic variations or immunological markers. Prediction and prevention, an increased efficacy, improved dosing and improved safety are some of the strengths of personalized medicine.

Some of the recent failures in stratified medicine [5] show the need for a better understanding of the molecular basis of diseases. However, the continuing advances in scientific knowledge will facilitate the move from the current stratified approach, which relies on static biomarkers of disease (with the exception of the new immunotherapies in cancer), to a truly individualized treatment, which considers the combination of dynamic biomarkers, disease heterogeneity in time, the ever changing environment, epigenetics and many other factors that modulate disease and response to treatment.

\section{PerMed Strategic Research and Innovation Agenda}

Under the FP7 programme, the European Commission funded a Coordination and Support Action on Personalized Medicine (PerMed). The main goals of the PerMed consortium were to complement existing activities in Europe by identifying and promoting promising research topics and bringing forward the implementation of personalized medicine for the benefit of society. The main output is the development of a Strategic Research and Innovation Agenda (SRIA) for Europe and beyond. The interaction of key European players was essential in this task and made it such a unique project. European and national decision-makers and funding bodies initiated this specific action to promote coordination and cooperation efforts between European key stakeholders showing their strong commitment to personalized medicine.

The methodology for the SRIA has been very rigorous, starting with an in-depth gaps-and-needs analysis of the available reports and literature, complemented by telephone interviews with key stakeholders in the different areas of public health and drug development. This was followed by workshops and conferences to discuss the results of the gaps-and-needs analysis, to facilitate multistakeholder discussions and to complete the SRIA. The SRIA was presented at a press conference in Brussels in June 2015 [2].

The results of the literature-based gaps-and-needs analysis have previously been published and will not be listed in this paper $[4,7]$. Figure 1 shows the main areas of concern identified in the analysis. In this paper, we concentrate on the area of the 'legal framework' and, more specifically, on the aspects of systematic early dialogue and early patient access (fig. 1, identified as the riskbased approach); future evaluations will also look at other aspects such as innovative clinical trial methodologies and designs. We evaluated the currently available tools in the EU and the USA. Furthermore, we made a systematic and detailed analysis of the conditional approvals (CAs) granted in the EU and the USA [named 'accelerated approvals' (AAs)] and the evidence accepted by regulatory authorities for this risk-based approach to marketing authorization. This analysis elucidates how prepared regulators are to accept early clinical evidence if it shows promising safety and efficacy data, and this can inform future discussions on the need to adapt regulations and approval pathways for personalized medicine.

\section{Early Patient Access/Risk-Based Approaches}

For marketing authorization, the benefit-to-risk ratio of a drug is evaluated. Only drugs with positive benefitto-risk ratios obtain authorization and are then available to patients to treat the approved indication (or to be used off label at the physician's discretion). In Europe, once marketing authorization is obtained, country-specific criteria are applied to decide whether drugs will be reimbursed within national health care systems and to set their prices; these criteria vary between relative effectiveness analysis (e.g. France, Germany), cost-effectiveness analysis (e.g. England) and other methods. 


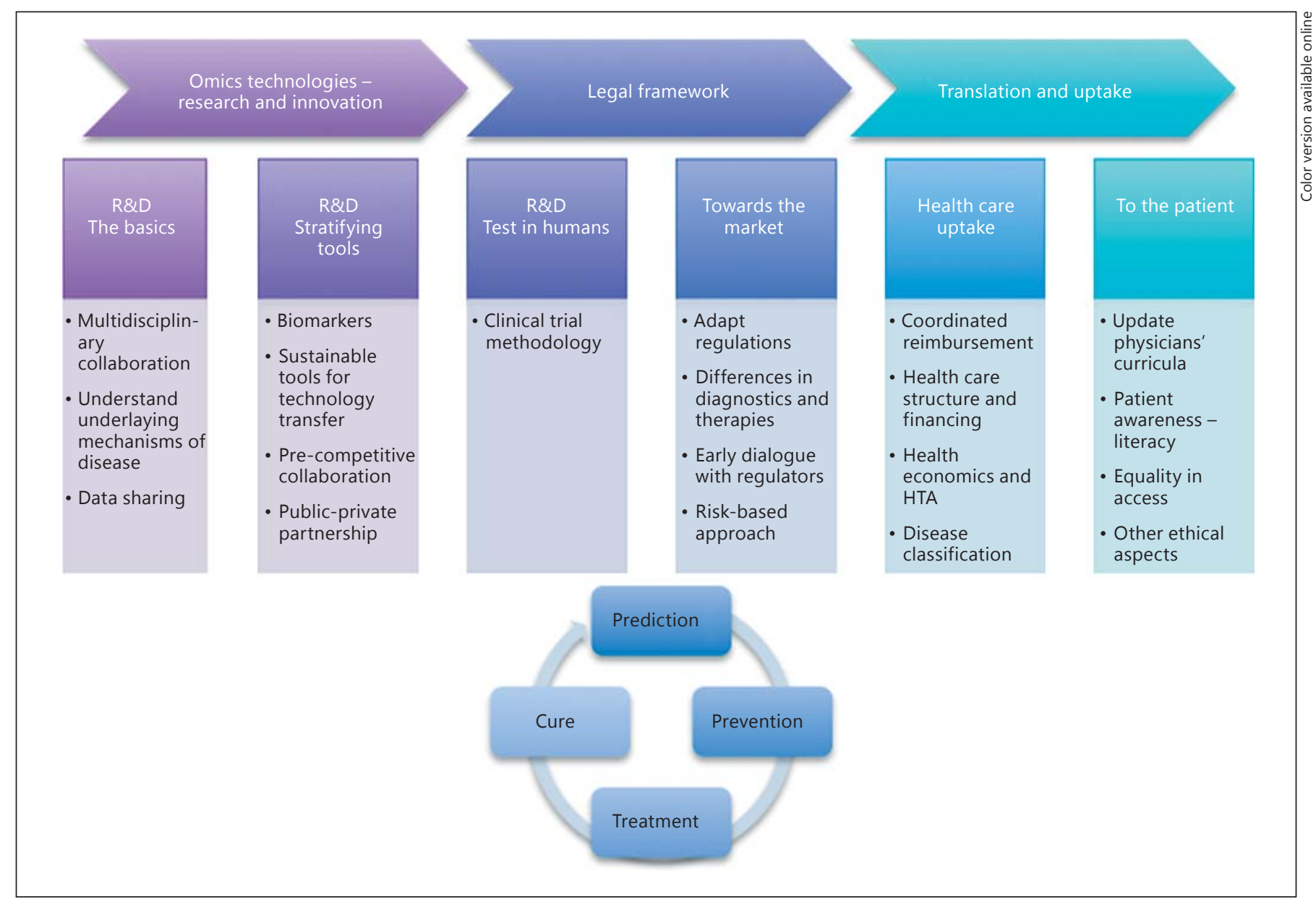

Fig. 1. Gaps-and-needs analysis for the personalization of medicine. Adapted framework from the European Commission [1] with the results from 18 reports on personalized medicine [2].

In the previous 10 years, we have seen the appearance of a number of regulatory tools that aim to facilitate earlier access of patients to efficacious and safe medicines. These tools can be either based on early, iterative and continuous dialogue or on risk-based marketing authorization pathways. By risk-based approaches, we refer to programmes that accept a higher degree of uncertainty if a drug shows very promising early efficacy results and an acceptable safety, leading to the sharing of risks between the regulator (and the general public) and the applicant. We present the different available tools in this section and evaluate the use of the CA process in the EU and the USA during the previous 5 years in detail in the next section. Table 1 and figure 2 present the different tools we identified to facilitate early patient access, differentiated by stage of regulatory evaluation and characteristic of the tool.

Early Patient Access to Medicines: EU and US Perspectives
Marketing Authorization (Benefit/Risk Evaluation)

Early and Continuous Dialogue

In addition to the well-established scientific advice process, whereby applicants approach regulators with specific questions on product development, there are new programmes and pilots available to facilitate a more continuous and iterative dialogue between regulators, applicants and other stakeholders during the whole drug development cycle. In the USA, drugs that treat unmet medical needs, either by providing a therapy for an indication for which there are no other drugs or by improving available therapies, can obtain fast track designation and are eligible for more frequent meetings and written communication with the Food and Drug Administration (FDA) to discuss the drug's development programme in addition to the rolling review of evidence. Breakthrough therapy designation is given to drugs that, based on preliminary clinical 
Table 1. Available regulatory tools for early dialogue and early patient access $[6,9]$

\begin{tabular}{llll}
\hline Stage of regulatory evaluation & Characteristic & EMA & FDA \\
\hline $\begin{array}{l}\text { Marketing authorization } \\
\text { (benefit/risk evaluation) }\end{array}$ & $\begin{array}{l}\text { Early and continuous } \\
\text { dialogue }\end{array}$ & Adaptive pathways & $\begin{array}{l}\text { Fast track } \\
\text { Breakthrough therapy designation }\end{array}$ \\
\cline { 2 - 4 } & Faster evaluation & Accelerated assessment & $\begin{array}{l}\text { Fast track } \\
\text { Priority review }\end{array}$ \\
\cline { 2 - 4 } & Less evidence & $\begin{array}{l}\text { CA } \\
\text { Exceptional circumstances }\end{array}$ & AA \\
$\begin{array}{l}\text { Market access } \\
\text { (relative effectiveness/ } \\
\text { cost-effectiveness evaluation) }\end{array}$ & $\begin{array}{l}\text { Early market access } \\
\text { (limited to EU market) }\end{array}$ & $\begin{array}{l}\text { Managed entry agreements } \\
\text { Cancer Drugs Fund (UK) }\end{array}$ & $\begin{array}{l}\text { Payers decisions, rebates and } \\
\text { negotiations (plus Medicare/ } \\
\text { EAMS (UK) }\end{array}$ \\
\hline
\end{tabular}

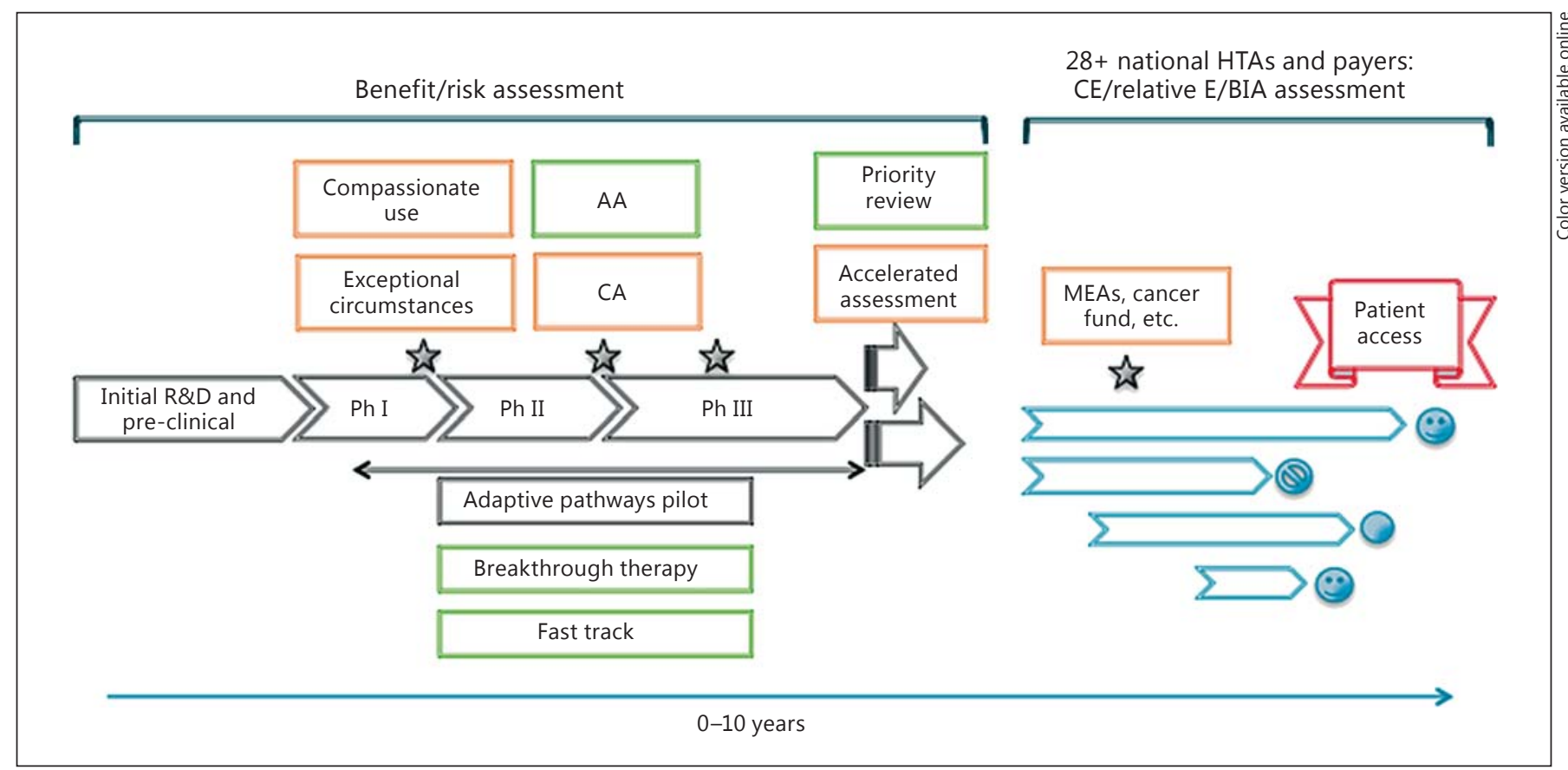

Fig. 2. Available regulatory tools for early dialogue and early patient access placed on the current drug development phases from initial R\&D to the market. Orange boxes: programmes available in the EU. Green boxes: programmes available in the USA. Colours refer to the online version only. $\mathrm{CE}=$ Cost-effectiveness; relative $\mathrm{E}=$ relative effectiveness; $\mathrm{BIA}=$ budget impact analysis; $\mathrm{MEAs}=$ managed entry agreements; $\mathrm{Ph}=$ phase. evidence, demonstrate substantial improvements over available therapies on clinically significant endpoints. The advantages are an intensive guidance to ensure an efficient drug development and an organizational commitment involving senior managers, in addition to all the advantages of the fast track designation. In Europe, the adaptive pathways pilot, launched by the European Medicines Agency (EMA) in 2014, also aims to offer a continuous dialogue from the early phases of development up to marketing authorization for therapies that fill an unmet medical need in order to encourage their efficient development, identify the best marketing authorization application (MAA) pathway and facilitate early patient access. The early involvement of health technology assessments (HTAs) and other stakeholders (including patients) is another important feature of this pilot. 
Table 2. Definitions of AA (FDA) [10] and CA (EMA) [11]

\section{AA programme}

Early approval based on markers that predict a reasonable benefit, with more testing to confirm a clinical benefit after approval. Allows early approval of a drug for a serious or life-threatening illness that offers a benefit over current treatments. This approval is based on a 'surrogate endpoint' (e.g. a laboratory measure) or other clinical measure the FDA considers reasonably likely to predict a clinical benefit of the drug. Once AA is granted, the drug must undergo additional testing to confirm that benefit; this speeds the availability of the drug to patients who need it.

\section{$C A$}

For certain categories of medicinal products, in order to meet unmet medical needs of patients and in the interest of public health it may be necessary to grant marketing authorizations on the basis of less complete data than is normally required. In such cases, a marketing authorization may be granted subject to certain specific obligations to be reviewed annually. This may apply to medicinal products for human use that belong to one of the following categories:

- medicinal products for seriously debilitating diseases or life-threatening diseases;

- medicinal products to be used in emergency situations, in response to public threats, and

- orphan medicinal products (drugs approved for small populations of patients with rare diseases).

Faster Evaluation Process Leading to Shorter

Assessment Periods

Faster review periods aim to translate into faster marketing authorization and faster patient access. The FDA priority review programme decreases the standard review time by 4 months (from 10 to 6 ), whereas the European accelerated assessment aims for a 2-month reduction (from the usual 210 evaluation days to 150 days excluding clock stops). We can also consider the FDA fast track designation under this category, since it offers the possibility of a rolling review of evidence instead of waiting for submission until the entire body of evidence is collected. Such a rolling process can potentially reduce the review time needed to process the full application. To incorporate this into the European regulatory landscape could prove challenging, since all national agencies are involved in the review of centralized authorization procedures.

When it comes to extensions of indication for one single agent, the evidence for its safety can in most cases be comparable to that for the existing indication (if the target population does not dramatically change and the inherent co-morbidities are not very different). Consequently,

Early Patient Access to Medicines: EU and US Perspectives the review of MAAs should be considerably shorter. One issue that could be considered in Europe is to introduce a mechanism that allows the submission of extensions of indications and new evidence during the review process of the initial MAA; this is possible in the USA and brings with it a considerable delay in the availability of drugs to patients for new indications (we acknowledge, however, that off-label applications are possible after the initial marketing authorization).

\section{Less Evidence (Risk-Based Approach regarding}

Evidence Requirements)

The early approval of medicines based on surrogate endpoints and less stringent evidence requirements has already been granted for a decade for drugs that offer very promising early results. These approvals are conditional on specific post-approval requirements to confirm the initial benefit/risk evaluation, including the need to do additional clinical trials or the finalization of ongoing trials. We will discuss the CA (EU) and AA (USA) processes in more detail in the next section and analyse their use during the previous 5 years in the USA and the EU, the indications for which they were granted, the time to approval and the level of evidence required.

\section{Market Access (Relative Effectiveness/ \\ Cost-Effectiveness Evaluation)}

Early Market Access after Marketing Authorization

Has Been Granted (EU Specific)

The schemes offer early market access and are mostly country and product specific. They facilitate patient access before relative effectiveness, cost-effectiveness or other criteria have been established. Managed entry agreements can be based on volume, prices or the results obtained by the medicine; some examples are risk-sharing agreements (used in France and Italy), payment by result (applied in Italy and Spain) or coverage with evidence development (used in the Netherlands and Sweden) [8]. They are usually individually negotiated between companies and authorities and are not transparent; furthermore, their benefits have not been systematically evaluated. The UK has just launched their new Early Access to Medicines Scheme (EAMS), whereby drugs can be made available to patients in the UK in advance of their eventual EU approval. The EAMS involves a two-stage procedure; the first stage involves gaining a promising innovative medicine designation, and the second stage involves gaining a positive scientific opinion from the Medicines and Healthcare Products Regulatory Agency (MHRA). The EAMS also encourages early parallel scien- 
Fig. 3. a EMA CAs per indication between 2007 and $2015(\mathrm{n}=17)$. b FDA AAs per indication between 2007 and $2015(n=25)$. c Median evaluation time (months).
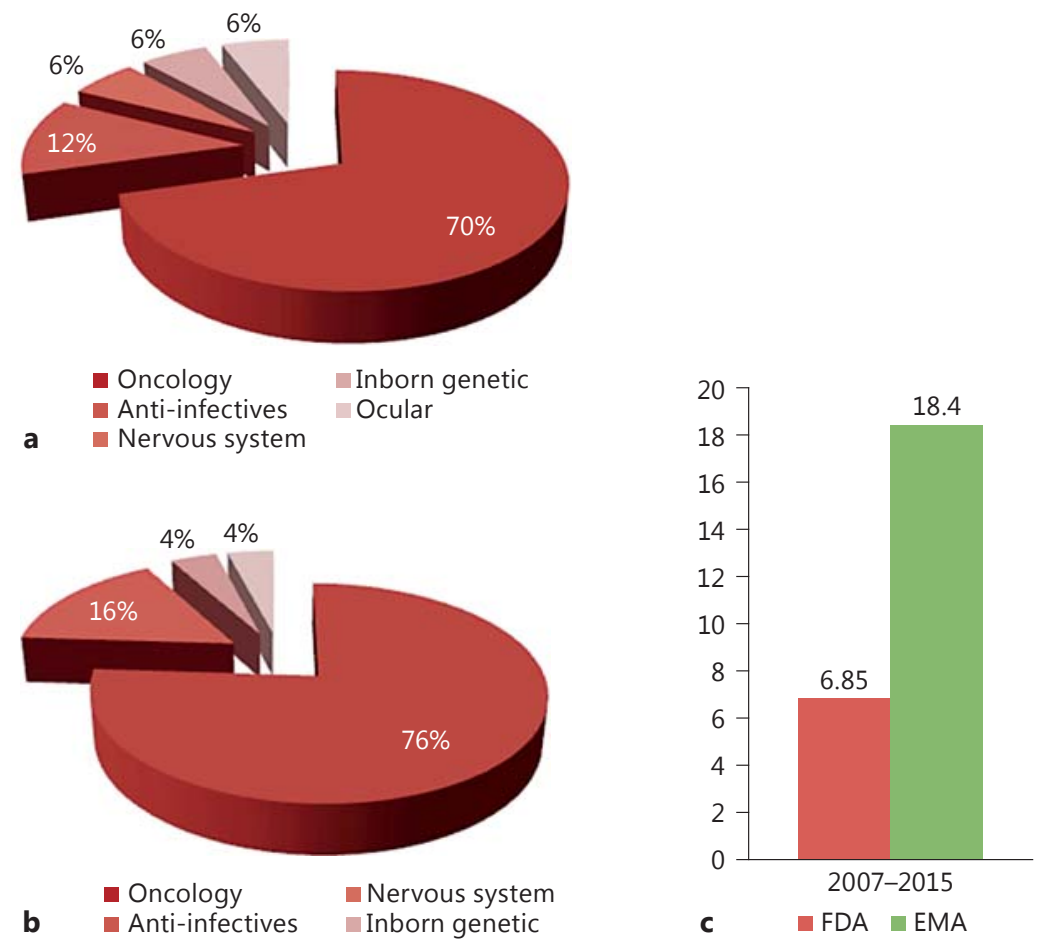

tific advice from the MHRA and the National Institute for Health and Clinical Excellence, analogous to the joint scientific advice offered by the EMA and HTAs.

\section{CAs from the EMA and AAs from the FDA}

CAs in the EU and AAs in the USA (table 2) are risksharing tools available to applicants to offer early patient access to innovative drugs if the applicants can prove with limited evidence from the early development phases (usually through surrogate endpoints) that their products are of major public health interest and fill an unmet medical need for serious conditions. If this is the case, it may be acceptable to have higher degrees of uncertainty in safety and efficacy and to grant marketing authorization based on post-approval conditions that confirm the initial, promising data. The EMA first published their guideline on conditional marketing authorization at the end of 2006; the FDA initiated their programme in 1992, but it was not incorporated into state regulation until it was passed by the Congress in 2012 (FDA Safety and Innovation Act) [9].

An in-depth analysis of the CAs granted by the EMA and the FDA (AA programme) is presented in this sec- tion. We have evaluated the CAs and AAs granted during the previous 8 years (2007-2015), and in more detail during the previous 5 years (2011-2015), based on public information published on the EMA and FDA websites. We analysed European public assessment reports (EMA) and summaries of reviews (FDA) to identify the products, the approved indications, the time to approval and the evidence submitted [12-16]. We only considered initial MAAs and did not look at further modifications, extensions or submissions for the same product; furthermore, in Europe only products approved through the centralized procedures were considered.

Between 2007 and the first half of 2015, 17 products received $\mathrm{CA}$ in the $\mathrm{EU}$ and 25 drugs received AA in the USA. As depicted in figure 3, most of the approvals were in the area of oncology, followed by anti-infectives and very few drugs for diseases of the nervous system, inborn genetic disorders (mostly enzyme replacement therapies) and ocular conditions (in the EU). There are only 4 medicines in common between the conditionally approved products in the EU and the USA (Zykadia, Sirturo, Adcetris and Xalkori); this may be due to differences in dates of submission to the EMA and the FDA: if submission to one of the two agencies is delayed by the applicant, more 
Fig. 4. a EMA CAs. Submitted evidence by phase (I-III) of pivotal CT 2011-2015. b FDA AAs. Submitted evidence by phase (I-III) of pivotal CT 2011-2015. c EMA CAs. Submitted evidence by design of pivotal CT 2011-2015. d FDA AAs. Submitted evidence by design of pivotal CT 20112015. $\mathbf{a}, \mathbf{c} \mathrm{n}=14 . \mathbf{b}, \mathbf{d} \mathrm{n}=20$. CT $=$ Clinical trial; $\mathrm{OL}=$ open label; $\mathrm{RRCT}=$ randomized controlled clinical trial; $\mathrm{UC}=$ uncontrolled.

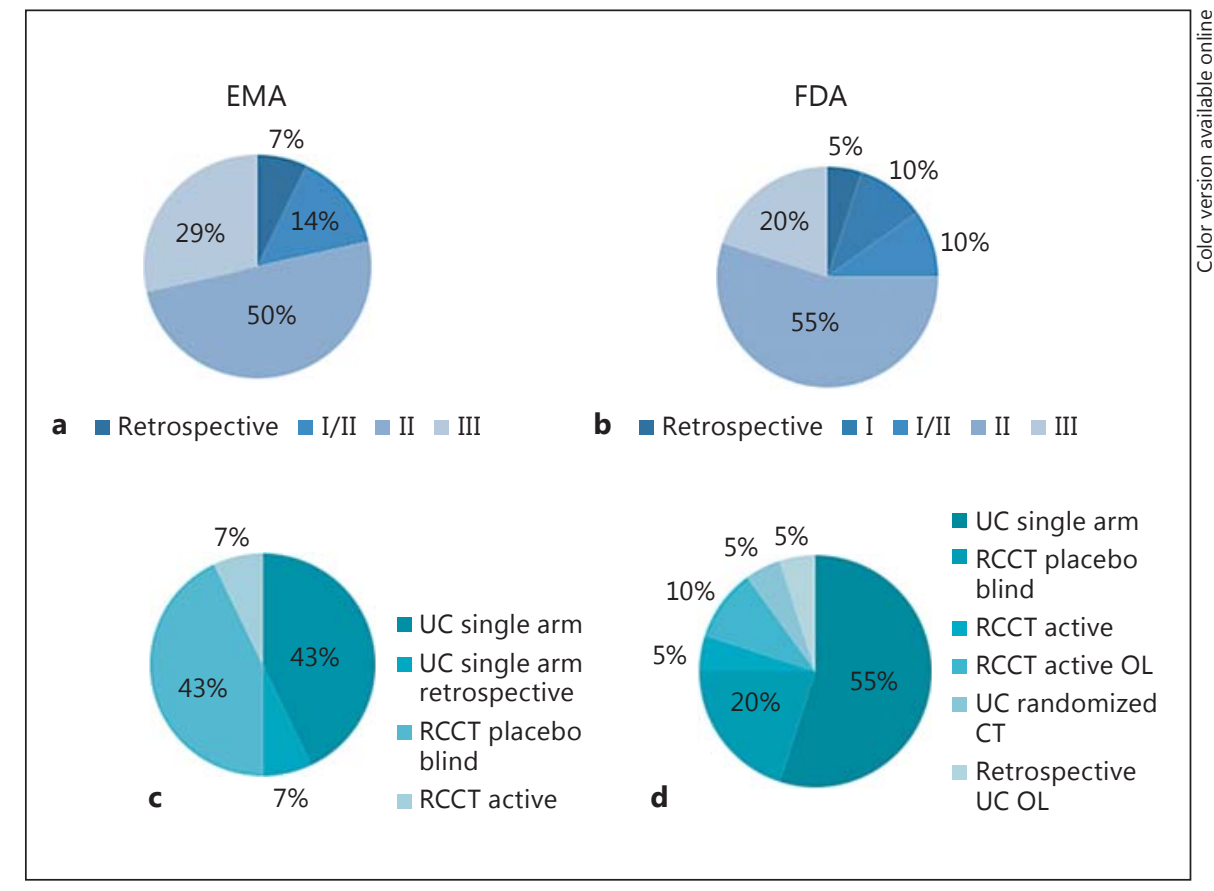

mature data may be available, offering the possibility of receiving full marketing authorization. Only $57 \%$ of the drugs approved in the EU had an orphan designation, compared to $95 \%$ in the USA. This may be due to the differences in the definition of orphan drugs (prevalence as defined in the EU is $\leq 5$ in 10,000 compared to $<200,000$ in USA) and the requirements for obtaining the orphan drug designation, or to the small overlap of compounds that obtained conditional approvals between both regions.

We will now concentrate on the type of evidence submitted to support marketing authorization accepted by the EMA and the FDA as being sufficient for CA and AA, respectively. Drugs approved during the 2011-2015 period were considered, i.e. 14 drugs in the $\mathrm{EU}$ and 20 in the USA. The distribution of products per indication does not significantly differ from that presented in figure 3 , except that the percentage of FDA AAs for oncology medicines increased to $84 \%$.

While the highest proportion of the medicines evaluated was approved based on pivotal data from phase II clinical trials (50\% in the EU and 55\% in the USA), retrospective data analysis was accepted as evidence for 1 drug in each region. These pivotal clinical trials were mostly uncontrolled single-arm trials (43\% in the EU and 55\% in the USA), followed by randomized placebo-controlled blinded clinical trials (fig. 4).

Fig. 5. a EMA CAs. Submitted evidence by number of trials 20112015. b FDA AAs. Submitted evidence by number of trials 20112015. c EMA CAs. Submitted evidence by number of patients 2011-2015. d FDA AAs. Submitted evidence by number of patients 2011-2015. a, $\mathbf{c ~} \mathrm{n}=14$. b, $\mathbf{d} \mathrm{n}=20$. 
Fig. 6. a EMA CAs. Submitted evidence by comparator type 2011-2015 ( $\mathrm{n}=14)$. b FDA AAs. Submitted evidence by comparator type 2011-2015 ( $n=20)$. c EMA CAs. Post-authorization requirements 2011-2015 ( $\mathrm{n}=17$; more than 1 category was requested for 3 products). d FDA AAs. Post-authorization requirements 20112015 ( $n=24$; more than 1 category was requested for 4 products).

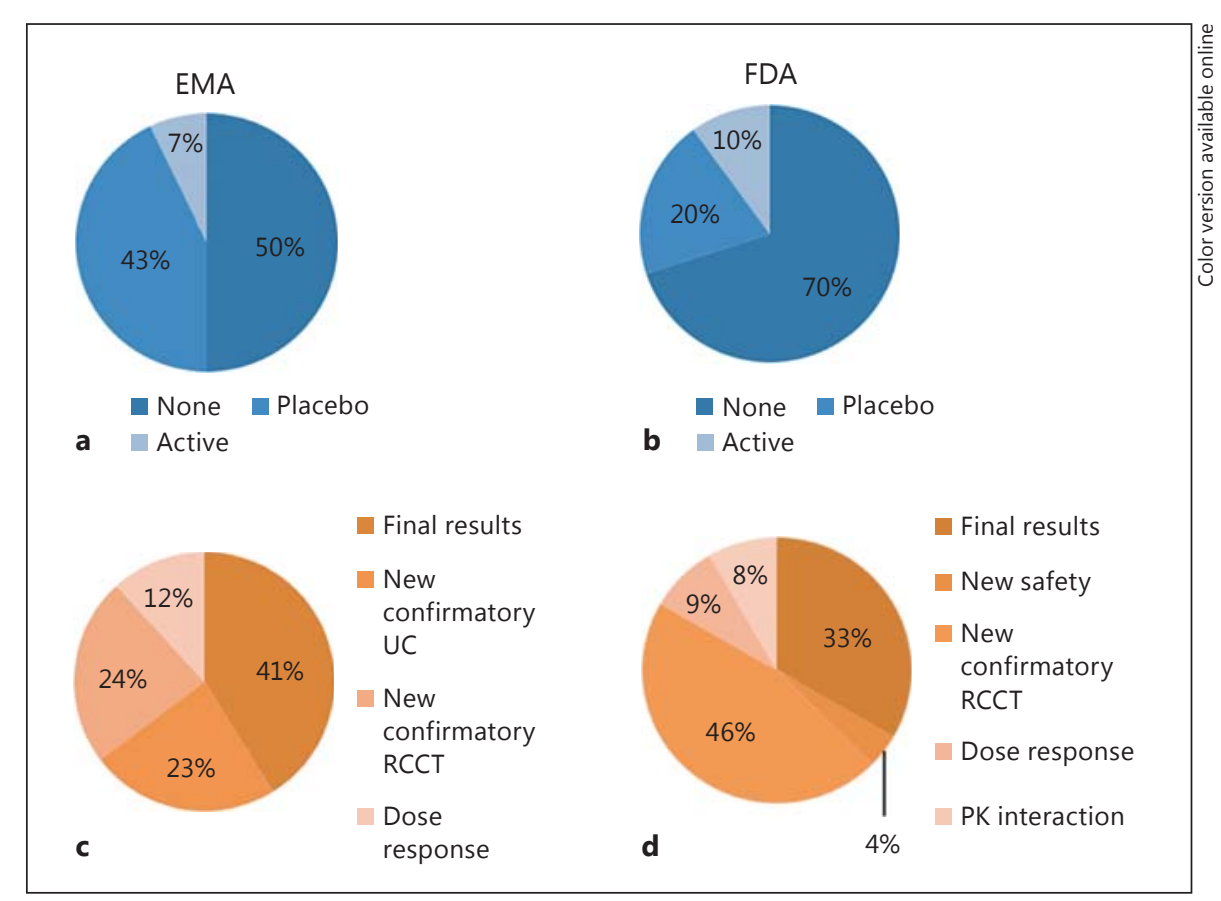

Most products in the USA were approved based on data from 1 or 2 clinical trials, whereas in the EU all products had data from at least 2 clinical trials. Another remarkable difference is the fact that all pivotal trials for the EU-approved products were at least phase II, while in the USA 2 products were approved based on data from phase I trials. The number of trials in figure 5 may be inflated for some products due to the fact that extension studies were considered equal to other clinical trials. The number of patients treated in clinical trials with the approved product for the approved indication also varied; most submissions included data from between 100 and 400 patients. Interestingly, some approvals were based on evidence from fewer than 100 patients, which is indeed expected for orphan drugs. The median number of patients was 245 (range 52-588) for EMA-approved products and 179 (range 84-456) for FDA-approved products.

Surprisingly, more than $50 \%$ of the products $50 \%$ in the EU and 70\% in the USA) were approved based on data from uncontrolled clinical trials. Efficacy and safety data from uncontrolled clinical trials represent higher degrees of uncertainty. In the controlled trials, placebo was the most common comparator, with very few products presenting comparisons with active comparators (fig. 6; 1 in the EU and 2 in the USA). FDA-approved products were all based on surrogate endpoints, whereas in the EU, only $64 \%$ of the products had efficacy evidence based on sur- rogate endpoints (they mostly correspond to oncology products, where mature overall survival data can take a long time to collect).

In terms of post-authorization requirements, completion of ongoing trials and reporting of final mature results were the most common requirements for the EMA-approved products, whereas the FDA more often requested new confirmatory randomized controlled clinical trials (fig. 6). Other requirements ranged from phase I trials to determine the correct dose and provide dose-response data, over pharmacokinetic interaction studies to new safety and uncontrolled clinical confirmation trials. We could not identify any trends in the post-authorization requirements that indicated a relationship between uncontrolled evidence and demands for new confirmatory controlled clinical trials; this may be due to the difficulty of conducting controlled trials in some orphan conditions.

\section{Implications for Personalized Medicine and a Forward Look}

In the previous section, we have seen how approval of medicines based on more limited evidence is possible when promising early results are available and there is a significant unmet medical need. Many experts in the field 


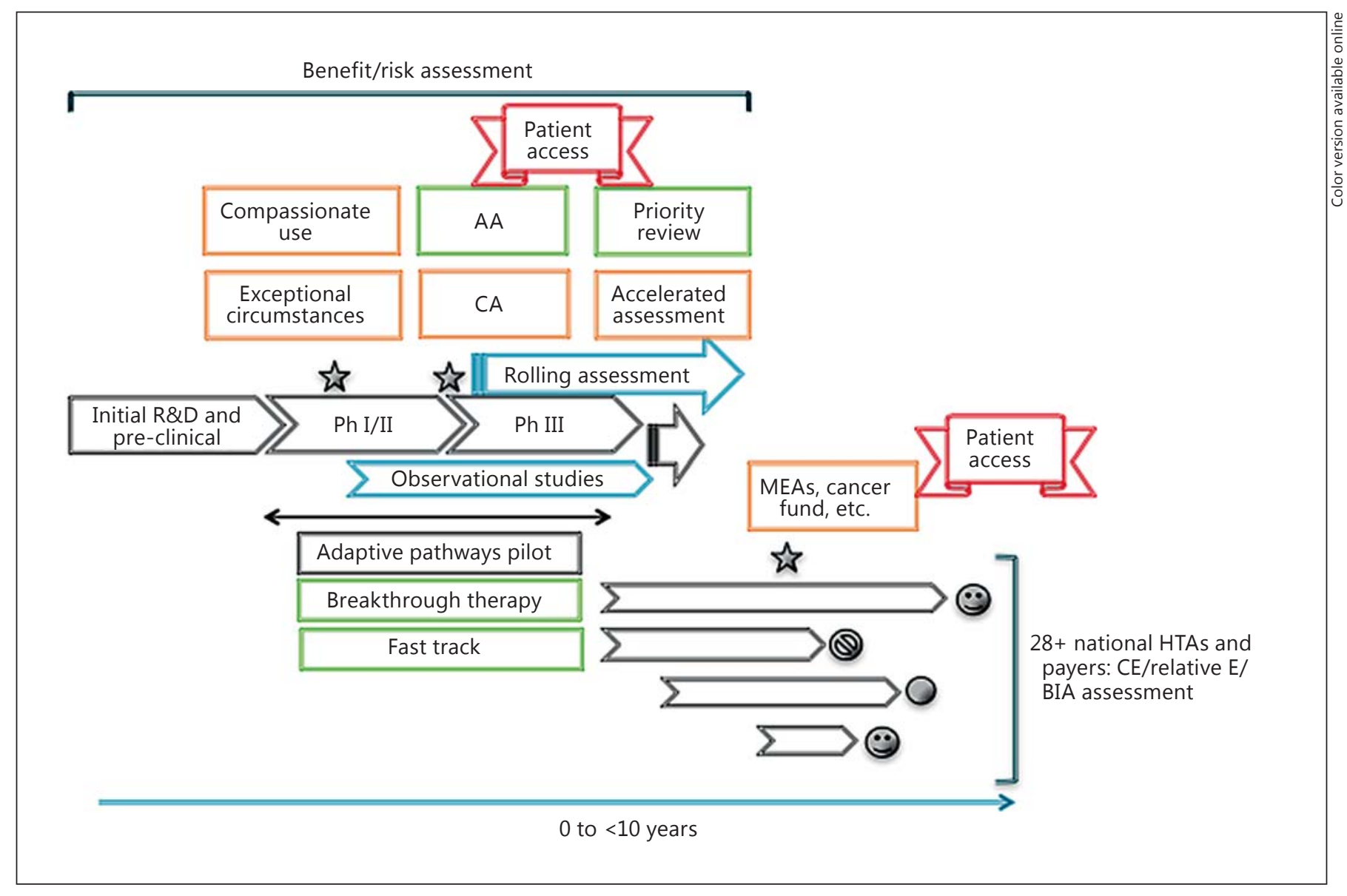

Fig. 7. Available regulatory tools for early dialogue and early patient access placed on the revised drug development phases from initial R\&D to the market. Orange boxes: programmes available in the EU. Green boxes: programmes available in the USA. Blue box- es: possible improvements. Colours refer to the online version only. $\mathrm{CE}=$ Cost-effectiveness; relative $\mathrm{E}=$ relative effectiveness; BIA = budget impact analysis; MEAs = managed entry agreements; $\mathrm{Ph}=$ phase. of personalized medicine are advocating for smaller and fewer clinical trials to speed up the development cycle and allow earlier patient access, and to some extent this is already the case. The high numbers of orphan and oncology drugs in our analysis support the view that smaller subsets of populations and smaller subsets of individual diseases are being characterized that previously have been considered as the same condition (e.g. we now know that the same mutation in tumours from different organs may bear more similarity than two tumours in the same organ with different mutations). As we move into smaller subsets of disease, more conditions could be associated with the 'orphan' definition. Already available drug development strategies for orphan conditions will be used more often; however, the topic of pricing has to be carefully reevaluated if we are to move into a new classification of diseases for many 'orphan' conditions, since current pricing tactics for orphan drugs would not be sustainable. Alternatively, as we move forward with new precision medicine being introduced, the expected impact in terms of unnecessary drug spending and reduced occurrences of drug adverse events should be introduced into the postauthorization assessment.

It is important to acknowledge that regulators in joint action with HTA bodies have to find the right balance between patients' need to access drugs faster and their inherent mission to protect patients from unsafe/inefficacious drugs. Therefore, a sufficient minimum amount of evidence is needed to ensure that these drugs are indeed safe and efficacious, and a certain time is needed to review the applications and clarify uncertainties with the applicants. Risk-based approaches to marketing authorization 
aim to share the risk of uncertainties in the benefit-to-risk ratio between applicants and regulators. In most cases, additional data are needed in order to confirm the preliminary conclusions on positive benefits and low risks.

The caveats of this analysis and proposals for future research will be presented here. To start with, only drugs were evaluated; however, different technologies (including medical devices) are used in medical practice and are becoming essential for personalized medicine, and differences in responsible regulatory authorities and regulations for drugs and medical devices in Europe pose additional challenges in this area that are outside the scope of this paper. Furthermore, only initial MAAs were considered; it could be interesting to evaluate further variations as well as the renewal of CAs and AAs, and whether the data submitted within the post-marketing requirements confirmed the initial results. There are some additional schemes facilitating early patient access that have not been discussed here, such as named patient access or compassionate use programmes for drugs in development. These can be linked to clinical trials, and physicians have to request special permission from regulatory authorities as well as the developer company for each individual patient. These schemes were excluded because they are not designed to reach the broader patient population and focus on individual patients. There may also be additional country-specific schemes that were not discussed here and could be evaluated in future analyses.

One issue, especially in European markets, is whether HTA bodies and other entities in charge of pricing and reimbursement would also accept the early and immature but promising evidence submitted for CA. HTAs may be hesitant to accept surrogate endpoints and immature data and could request additional data collection, delaying the time to market. In such cases, CAs and other risk-based approaches pioneered by regulators may not result in the earlier patient access aimed at if the bottleneck to market is at the latter stage of market access. However, the emerging developments in the direction of an improved coordination and alignment of regulatory processes and HTAs may provide a future framework for handling these challenges. This issue has to be thoroughly considered and discussed to find solutions able to balance earlier patient access with sufficient evidence for a therapeutic value with reference to clinical effect and safety. To approach these challenges, it appears relevant to take into consideration the use of appropriate tools for monitoring novel pharmacological agents under real-world conditions. (analogous to fig. 2) with some modifications to incorpo-

rate a scenario where the tools described in this paper are widely used and early dialogue is the norm. The incorporation of observational studies parallel to later stages of clinical development (as the adaptive pathways pilot aims to promote) to inform final marketing authorization after CAs - or the establishment of a rolling assessment of evidence and a compulsory early and continuous dialogue for promising drugs in the EU (as in the FDA) - would be some of the possible ways to improve the system.

From the presented analysis and the regulatory tools for facilitating patient access, we can infer that regulators are indeed starting to accept early clinical evidence if it shows a promising safety and efficacy (as early as in phase I data). Some of the tools may not be used as much as they should, since most of them are voluntary and have to be requested by the applicant. Some of the tools and programmes described may need more refinement, or to be replaced by more appropriate ones, whereas others still need to be evaluated by the regulator (e.g. adaptive pathways pilot). Before strongly arguing for a complete change in regulations, we need to analyse what is available and where it could be improved. Furthermore, change can only happen step-wise; drug development and approval is an ongoing process, with many products in the pipeline that cannot be changed in a very short time span. Finally, and most importantly, we should remember that most regulations in this area had followed huge public health disasters jeopardizing the health of thousands of people (e.g. thalidomide), and, indeed, patients' safety and wellbeing should be in the foreground in all stakeholders' agendas.

\section{Disclosure Statement}

The authors confirm that they do not have any conflicts of interests or any financial interests. Furthermore, the views expressed in this publication are the views of the authors and might not represent the views of the institutions they represent.

Figure 7 presents the pathway from bench to market

References
Public Health Genomics 2015;18:249-259 DOI: $10.1159 / 000437137$
1 European Commission: Use of '-omics' technologies in the development of personalised medicine. Brussels, European Commission, 2013.

2 PerMed: Shaping Europe's Vision for Personalised Medicine. Strategic Research and Innovation Agenda (SRIA). Cologne, German Aerospace Center (DLR), 2015. http://www. permed2020.eu/_media/PerMed_SRIA.pdf.

3 Munoz J, Kurzrock R: Targeted therapy in rare cancers - adopting the orphans. Nat Rev Clin Oncol 2012;9:631-642. 
4 Leyens L, et al: Working towards personalization in medicine: main obstacles to reaching this vision from today's perspective. Pers Med 2014;11:641-649.

5 Berndt ER, Trusheim MR: The segmentation of therapeutic populations in oncology. Health Manage Policy Innovation 2012;1:1934.

6 European Medicines Agency. http://www. ema.europa.eu/ema/ (accessed June 15, 2015).

7 Horgan D, Jansen M, Leyens L, et al: An index of barriers for the implementation of personalised medicine and pharmacogenomics in Europe. Public Health Genomics 2014;17: 287-298.

8 Ferrario A, Kanavos P: Managed entry agreements for pharmaceuticals: the European experience. Brussels, EmiNET, 2013.
9 US Food and Drug Administration: Fast track, breakthrough therapy, accelerated approval, priority review. http://www.fda.gov/ ForPatients/Approvals/Fast/default.htm (accessed June 15, 2015).

10 US Food and Drug Administration: New drugs at FDA: CDER's new molecular entities and new therapeutic biological products. http://www.fda.gov/Drugs/DevelopmentApprovalProcess/DrugInnovation/default.htm.

11 European Medicines Agency, Committee for Medicinal Products for Human Use: EMEA/509951/2006: guideline on the conditional marketing authorisation for medicinal products for human use. London, European Medicines Agency, 2006.

12 European Medicines Agency: European public assessment reports. http://www.ema.europa.eu/ema/index.jsp?curl=pages/medicines/ landing/epar_search.jsp\&mid=WC0b01 ac058001d124 (accessed June 15, 2015).
13 US Food and Drug Administration, Center for Drug Evaluation and Research: Novel New Drugs 2011. Washington, US Food and Drug Administration, Center for Drug Evaluation and Research, 2012.

14 US Food and Drug Administration, Center for Drug Evaluation and Research: Novel New Drugs 2012, Summary. Washington, US Food and Drug Administration, Center for Drug Evaluation and Research, 2013.

15 US Food and Drug Administration, Center for Drug Evaluation and Research: Novel New Drugs 2013, Summary. Washington, US Food and Drug Administration, Center for Drug Evaluation and Research, 2014.

16 US Food and Drug Administration, Center for Drug Evaluation and Research: Novel New Drugs 2014, Summary. Washington, US Food and Drug Administration, Center for Drug Evaluation and Research, 2015. 